\title{
Network convergence and QoS for future multimedia services in the VISION project
}

\author{
Luis Pérez ${ }^{\mathrm{a}}$, Luis Velasco ${ }^{\mathrm{b}, *}$, Juan Rodríguez ${ }^{\mathrm{a}}$, Pedro Capelastegui ${ }^{\mathrm{c}}$, Guillem Hernández-Sola ${ }^{\mathrm{b}}$, \\ Lorena Calavia ${ }^{\mathrm{d}}$, Antonio Marqués ${ }^{\mathrm{a}}$, Borja Iribarne ${ }^{\mathrm{a}}$, Amador Pozo ${ }^{\mathrm{e}}$, Antoine De Poorter ${ }^{\mathrm{f}}$ \\ ${ }^{a}$ Telefónica $1+D$, Madrid, Spain \\ ${ }^{\mathrm{b}}$ Universitat Politècnica de Catalunya (UPC), Barcelona, Spain \\ ${ }^{c}$ Universidad Politécnica de Madrid (UPM), Madrid, Spain \\ ${ }^{\mathrm{d}}$ Universidad de Valladolid (UVA), Valladolid, Spain \\ e TELNET Redes Inteligentes, Zaragoza, Spain \\ ${ }^{\mathrm{f}}$ Ericsson España, Madrid, Spain
}

Keywords:

Network convergence

Multimedia services

Quality of service

\begin{abstract}
A B S T R A C T
The emerging use of real-time 3D-based multimedia applications imposes strict quality of service (QoS) requirements on both access and core networks. These requirements and their impact to provide end-to-end 3D videoconferencing services have been studied within the Spanish-funded VISION project, where different scenarios were implemented showing an agile stereoscopic video call that might be offered to the general public in the near future. In view of the requirements, we designed an integrated access and core converged network architecture which provides the requested QoS to end-to-end IP sessions. Novel functional blocks are proposed to control core optical networks, the functionality of the standard ones is redefined, and the signaling improved to better meet the requirements of future multimedia services. An experimental test-bed to assess the feasibility of the solution was also deployed. In such test-bed, set-up and release of end-to-end sessions meeting specific QoS requirements are shown and the impact of QoS degradation in terms of the user perceived quality degradation is quantified. In addition, scalability results show that the proposed signaling architecture is able to cope with large number of requests introducing almost negligible delay.
\end{abstract}

\section{Introduction}

In the recent years, personal communication systems have appeared, mostly based on the wide usage of the Internet. Nevertheless, as a consequence of the lack of reliable communication systems with the capacity to convey a real presence feeling, people continue travelling to attend work meetings. Multimedia services remain in 2D which prevents users from experiencing immersive tele-presence sessions. Aiming at improving user experience, stereo- scopic video (also known as 3D video) is already popular in movie theaters. Technologies based on 3D are currently reaching the consumer electronics market in devices such as TV sets, game consoles, and handheld mobile terminals. However, services such as $3 \mathrm{D}$ presence, videoconference, or on-line gaming present really strict QoS requirements, not only regarding high bandwidth, but also very low delay. Operators have to manage these requirements in the access as well as in the core network as a consequence of the significant need of resources.

In this context, since adding advanced 3D video features into multimedia sessions is a very promising area for network operators and vendors, a Spanish funded project named VISION was devised and carried out [1]. The project 
pursued the development of a 3D videoconference system, with a strong feeling of physical presence, over a data network. The developed system is able to reproduce real size, color, and depth of the users by means of a real-time multicamera/microphone capture of the local scene.

From the network viewpoint, the VISION architecture is based on the Telecommunications and Internet converging Services and Protocols for Advanced Networking (TISPAN) architecture for Next Generation Networks (NGN) [2]. An overview of the standardization process to define a QoS control architecture is presented in [3]. A TISPAN key subsystem is the IP Multimedia Subsystem (IMS), mainly based on the 3rd Generation Partnership Project (3GPP) [4]. The VISION project integrates a 3GPP IMS as well as an Automatically Switched Optical Network (ASON) [5] provided with a Generalized Multiprotocol Label Switching (GMPLS) control plane [6], hereafter referred as Wavelength Switched Optical Network (WSON). To this end, new modules have been defined to control optical resources and to aggregate IP flows in the existing optical connections, establishing new ones only when they are strictly needed. The proposed functional blocks are able to request for optical connection set up and teardown through a UserNetwork Interface (UNI) [7]. Moreover, NGN-based multimedia applications, such as $3 \mathrm{D}$ videoconferencing, require an enhanced control plane to be supported, features that nowadays are not, to the best of our knowledge, included in neither TISPAN nor 3GPP guidelines.

Some other works can be found in the literature regarding, in part, the topics tackled in the networking part of the VISION project. To start with, works [8-10] propose using the Session Initiation Protocol (SIP) [11] for on-demand session management. The authors in [8] design, analyze, and study the performance of a SIP-based videoconferencing desktop client. Additionally, a guideline for managing SIP-based services is also provided. Authors in [9] propose to add a session control plane, implemented using SIP, to optical burst switching networks so to close the gap between application requests and network control. Finally, in [10] an experimental validation of a service oriented optical network architecture based on SIP is presented. Although in our approach SIP is also used to establish user sessions, our network architecture includes an IMS for session control. Moreover, our network includes not only an optical network but also an IP network and a fiber-tothe-home (FTTH)-based access network.

Since one of the mayor concerns is as regards the scalability of the IMS, some works can be found in the literature focused on that issue (e.g. [12-15]). Authors in [12] provide guidance for constructing a robust and scalable IMS. For scalability and redundancy purposes, multiple SIP proxies and Application Servers (AS) are usually deployed. Then, to minimize delays the number of subsystems involved in the signaling path for a given user should be limited. A different mechanism to improve the scalability of SIP proxies increasing the overall request throughput is presented [13], where the authors propose to dynamically distribute the state across multiple nodes in contrast to stateful or stateless configurations. Note that in the context of the VISION project, long signaling paths are not an issue since few elements take part of SIP signaling as detailed in
Section 3. Aiming at providing useful information regarding IMS implementation and deployment, authors in [14] present an implementation of a distributed conferencing framework compliant with the IMS specification. Note that distributed conference is more suitable in multi-domain scenarios, showing better scalability than centralized conference. In this regard, the VISION architecture has been devised following the centralized approach to simplify video processing and QoS architecture, issues not tacked in [14]. Authors in [15] present a prototype of IMS-based presence service for multi-domain mobile scenarios, where dealing with heterogeneous wireless networks is a big challenge. They present some interesting proposals for scalability and quality optimization, such as a federated model to optimize inter-domain distribution of notification messages and differentiated quality levels. Nevertheless, these proposals optimize the exchange of subscription and notification messages, so they cannot be easily translated to the setup of multimedia sessions, which is the focus of the VISION project.

QoS degradation highly impacts on the users perceived service quality, also known as Quality of Experience (QoE) $[16,17]$. Besides QoS, a number of different factors are included in the QoE evaluation, such as the Grade of Service (GoS), and the quality of resilience [18]. Nevertheless, our focus in this paper is primarily on QoS. Our approach consists in guaranteeing enough resources to convey user flows and designing a per-flow set of priorities to allow flow preemption, in opposite to the per-packet approach proposed in [19]. These priorities are integrally managed by the transport network and installed as QoS rules in the IP routers. Moreover, low delay is achieved as a result of the technologies used in the network. The impact of this strategy is experimentally analyzed from the users perceived quality viewpoint.

Regarding multiparty services over converged networks, authors in [20] describe extensions to the session control procedures in the IMS to support multicast based multi-user services. GoS is evaluated both analytically and experimentally and the results are compared with standardized values. In contrast, our approach is based on a unicast IP network, where flow replication is performed by a Multipoint Conference Unit (MCU). We propose extensions to the standards regarding conference events and new blocks and interfaces for network control. In addition, we rely on ensuring QoS parameters to guarantee excellent perceived service quality. Finally, an experimental demonstration of the proposed extensions, functional blocks and architecture has been deployed, thus allowing for experimental validation and performance evaluation.

The remainder of the paper is organized as follows. Section 2 provides a general background starting with an overview of the VISION project and the considered multimedia scenarios followed by a brief description of the network convergence standards developed both by TISPAN and 3GPP. Section 3 translates the considered scenarios to requirements to be met by the transport network. Then, our approaches for session negotiation, network architecture, and service priorities are presented. New extensions and new blocks and interfaces are described to meet the 
strict QoS requirements. Section 4 describes the project deployment and the tests performed to quantify the perceived service quality and scalability. Finally, Section 5 concludes the paper.

\section{Background}

\subsection{Overview of the VISION project}

A large consortium consisting of leading Spanish companies and research centers and universities carried out the VISION project from 2007 till early 2011. Its main objective was the development of an immersive videoconferencing system giving users the sensation of presence and involved the following main functional working areas:

- Scenes capture: with the challenge of acquiring large scene information synchronously in real-time.

- Codification, aggregation and distribution: these topics deal with receiving multimedia data synchronously at reception. Video data is coded as defined in H.264 [21] with a pool of hardware encoders before streaming and hardware decoded at reception.

- Interactive real-time communications: this working area is aimed at providing QoS mechanisms in the network to be used by the videoconferencing system flows, and it is the main focus of this paper.

- Scene reconstruction: once multimedia data arrive at the destination, several real-time algorithms process both video and audio. Video can be displayed in 2D or 3D terminals. Audio is played using a speakers array. Moreover, a direction of arrival estimation system computes the user's position, so it is considered when presenting the video and audio flows.
- Human factors: sensation of presence and user experience factors have been studied in the project from the psychology viewpoint and their conclusions allowed enhancing system features.

At the end of the project, a multiuser system with many extra features, such as recording service, presence detection, gestural interaction, advance conference scheduling, 3D audio rendering, etc., was available. Fig. 1 shows an overview of the system.

The integrated VISION videoconferencing system was put into practice under three different scenarios: residential, entertainment, and corporate.

The residential scenario was designed to offer service for informal communication at home with low hardware requirements. This scenario needs only two cameras which are placed in the front side of the user, in addition to one encoder, one decoder, and the audio system. Three different video signals, encapsulated into one traffic flow, are sent: the left and the right images together with a depthmap. Additionally in this scenario, remote users can also share different pictures in a photolog.

The entertainment scenario provides a high quality videoconference service with new features based on 3D reconstruction. For tests, a virtual gym class was built, consisting of two different roles: a "trainer" who shows different exercises to remote "trainees". Trainees capture system is analogous to that in the residential scenario (two frontal cameras) so the trainer visualizes all the trainees in a grid on a single display. On the contrary, the trainer's room is equipped with 18 different cameras in addition to the frontal ones. This arrangement allows trainer 3D reconstruction to be displayed into a virtual scenario, e.g. a Japanese garden.

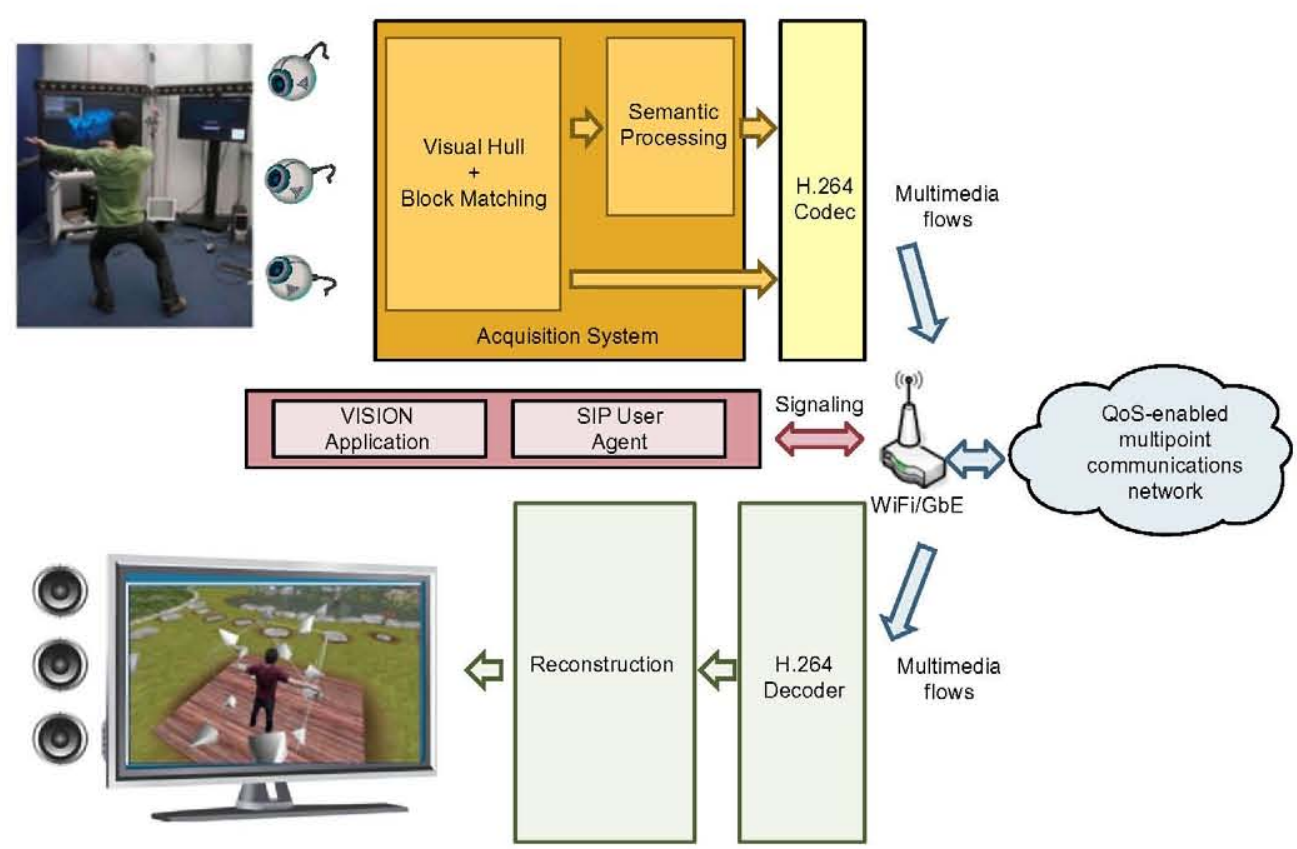

Fig. 1. Overview of the immersive videoconferencing system. 
The business scenario involving videoconference for companies is called corporate. In this scenario, users are displayed in a fixed position in a virtual meeting table which is placed in a virtual meeting room. Although only four views are used to render each user, user rooms are identical to that of the trainer in the entertainment scenario, i.e. 20 cameras, thus allowing each user to be rendered in different table positions chosen by remote users.

\subsection{Network convergence standards}

As aforementioned, IMS is an architectural framework for delivering IP multimedia services, originally designed by the 3GPP and later embraced by other standardization bodies such as ETSI TISPAN. From a network perspective, IMS is an architecture based on industry standard interfaces and protocols to control and deliver multimedia services. Its architecture is separated into three planes; from the top to the bottom we find the application, control, and transport planes. The application plane provides an infrastructure for the provision and management of services. The control plane routes the session signaling, announces the type of traffic to the transport plane, and generates the billing information. Finally, the transport plane provides a core QoS-enabled network with access from User Equipment (UE) over fixed or mobile networks.

IMS re-uses industry standard protocols, such as SIP to establish voice and multimedia sessions; SIP is based on a client-server model. Additionally, SIP is complemented by the Session Description Protocol (SDP) [22] and by the Real-time Transport Protocol (RTP) [23]. SDP is used to describe the multimedia stream to be established whereas RTP is used for the real-time multimedia transport. Another important protocol used in IMS is Diameter [24] which consists of a base protocol specification which may be extended by each Diameter specific application.

The architecture of the IMS consists of several interconnected functional blocks. Fig. 2 depicts a simplified scheme of the IMS architecture. The Home Subscriber Server (HSS) is a central repository containing subscriber related information. The Call Session Control Function (CSCF) deals with the SIP signaling. It consists of three different modules: Proxy-CSCF (P-CSCF), Interrogating-CSCF (I-CSCF), and Serving-CSCF (S-CSCF). The P-CSCF is a SIP Proxy which is the first entry point of the user to the IMS network. It provides secure signaling and acts as the interface with the network policy infrastructure for media flow authorization. The S-CSCF module communicates with HSS during the registration and authentication process, routes SIP messages to one or more ASs, and handles multimedia sessions. Moreover, the Media Resource Function (MRF) provides multimedia functions to establish multimedia flows in the network. The MRF actually consists of two functions: the controller (MRFC) that acts as a SIP User Agent (UA) receiving SIP signaling from the S-CSCF, and the processor (MRFP) that deals with multimedia flows. Finally, each different AS provides specific services to the end users, such as multimedia telephony.

In addition to the IMS architecture, 3GPP defines the generic functionalities for Policy and Charging Control (PCC) [25], linking control and transport planes. Its functionality encompasses: flow-based charging and policy

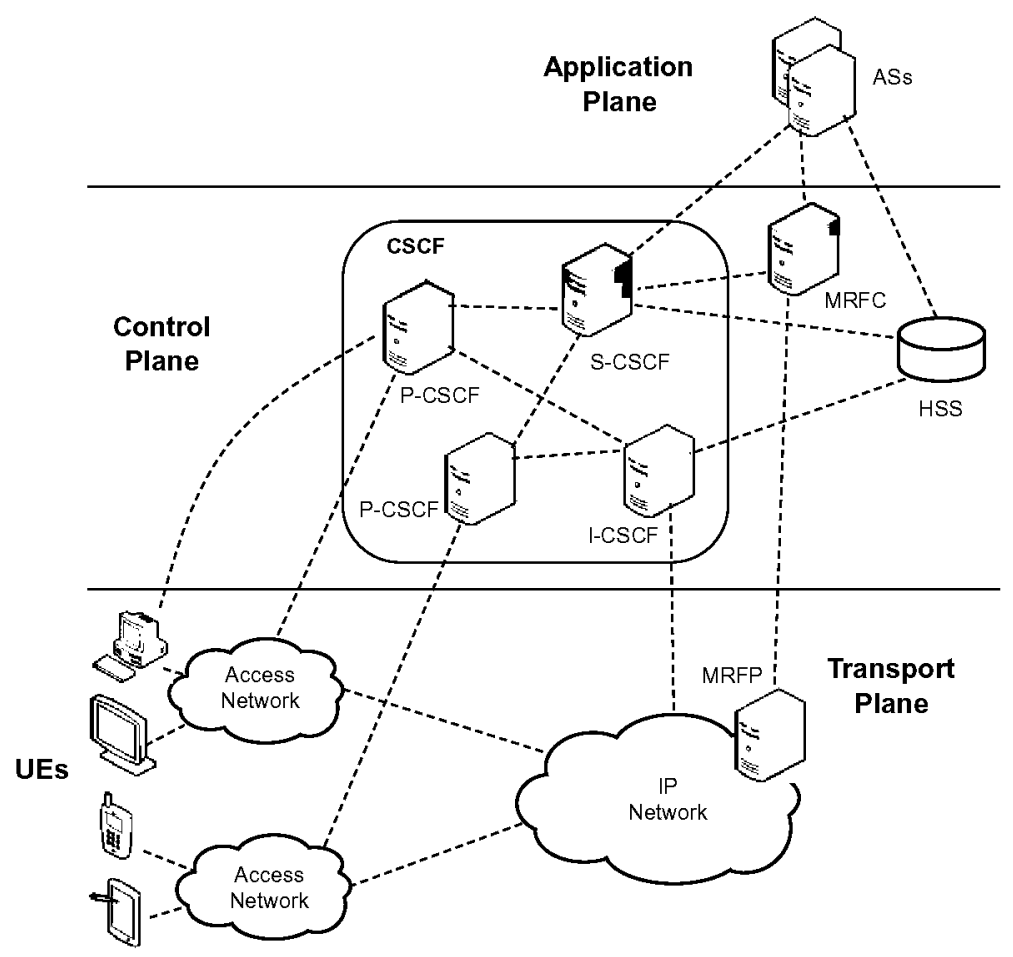

Fig. 2. Simplified IMS architecture as defined by $3 G P P$. 
control e.g. QoS control. The PCC element includes the Policy Control and Charging Rules Function (PCRF) which provides network control and flow-based charging towards the Policy Enforcement Function (PCEF). The PCRF receives through the $\mathrm{Rx}$ interface session and media related information from P-CSCF and informs it about traffic plane events. The PCRF provisions PCC Rules via the Gx interface.

In TISPAN, however, the control and transport plane linkage entity is called the Resource and Admission Control Subsystem (RACS) [26]. The RACS subsystem controls the status of transport network resources to guarantee the appropriate QoS level. It consists of two parts: the Service Policy Decision Function (SPDF) and the Resource and Admission Control Function (RACF). RACF receives requests from the SPDF, using the received QoS information to perform admission control. Then, it indicates SPDF whether a request for resources is granted or not.

Although different interfaces have been defined in TISPAN between P-CSCF and SPDF (Gq') as well as between SPDF and RACF (Rq), they are similar, but not identical, to $\mathrm{RX}$ and GX, respectively as defined in 3GPP. That can be also extended to blocks; although the specified blocks are different between TISPAN and 3GPP, general functionalities are quite similar in both standards which open up the opportunity for mixing blocks and interfaces creating novel hybrid approaches that may better fulfill specific requirements.

\section{Transport plane design}

\subsection{Requirements}

Fig. 3 shows a simplified scheme of the VISION architecture. It includes the transport (plane) network, the VISION terminals, a MCU, and the VISION Conference AS.

Each VISION terminal consists of a set of modules coordinated by a central element called VISION Application. A SIP UA is responsible for session signaling whereas media flows exchanged are managed by a codec. The VISION terminal includes video capture and a display. Finally, a graphical interface has been implemented to manage the terminal.

The VISION Conference AS allows advanced conference resource reservation for any of the scenarios considered in the VISION project. The MCU is responsible for providing the multiparty functionality required for some of the considered scenarios. It performs the role of a MRF in the 3GPP architecture and, as such, it is divided into a controller (MRFC), which manages SIP signaling, and a processor (MRFP) which is in charge of all media processing including multimedia flow replication.

Tables in Annex 1 define the flows to be transmitted from one user to the MCU and from the MCU to the rest of users, for the residential, entertainment, and corporate scenarios. As observed, $4.8 \mathrm{Mbps}$ and 4 flows are needed for each user in the residential scenario, around $19.2 \mathrm{Mbps}$

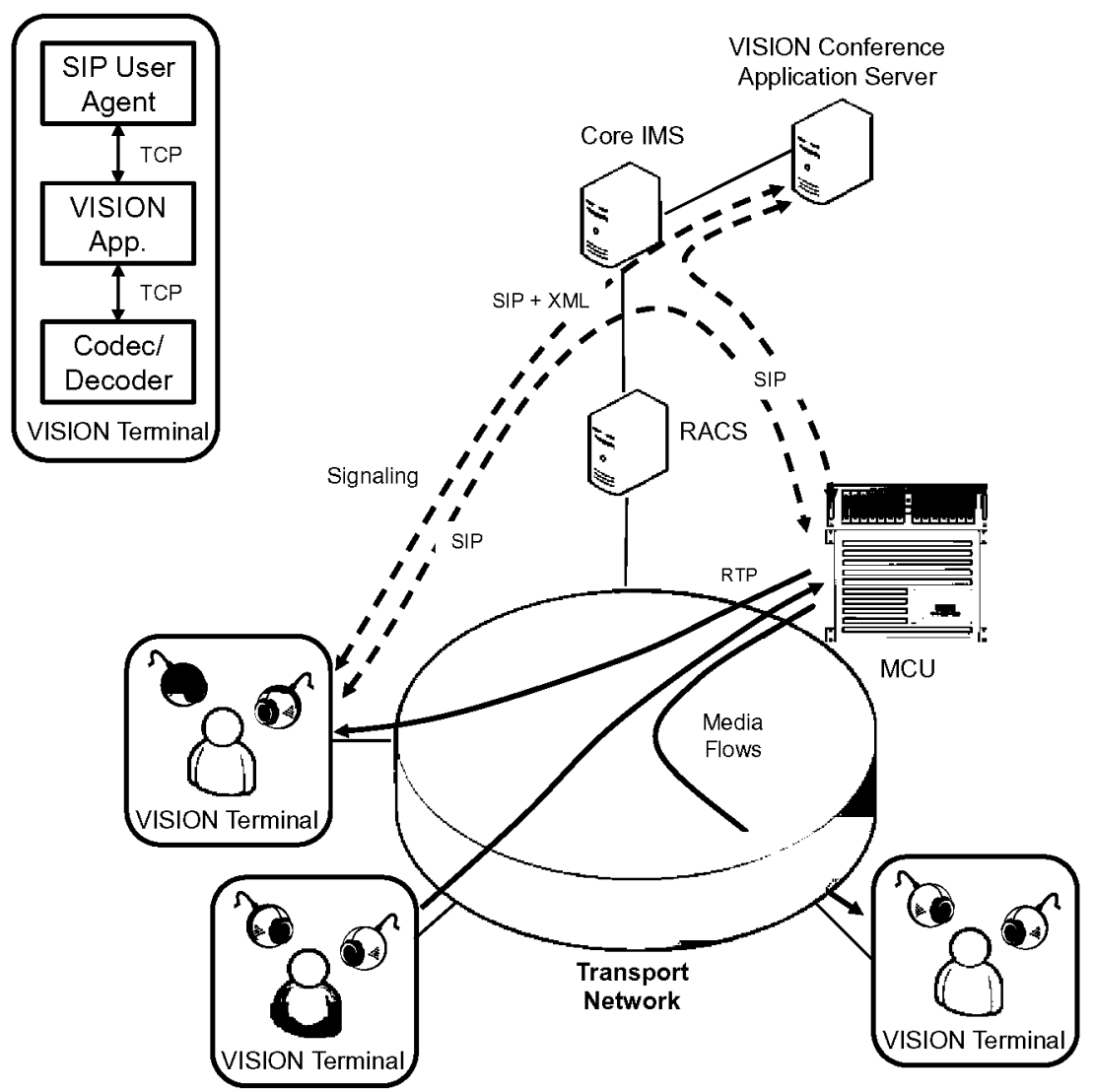

Fig. 3. The VISION architecture. 
and 23 flows are needed for the trainer, and $4.8 \mathrm{Mbps}$ and 4 flows for each trainee in the entertainment scenario, and around 19.2 Mbps and 23 flows are needed for each user in the corporate scenario.

\subsection{Session negotiation}

Media negotiation in SIP sessions begins with the calling SIP UA sending an initial session description with a series of options for media configuration. The called UA answers back with its preferred choices. After this initial exchange, further negotiation steps can update the session configuration if needed.

However, session establishment in VISION involves negotiating a higher number of parameters than for a typical video conference. Not only the large amount of media streams need to be configured, as a result of multiple cameras per site and the additional data flows, but also further information such as camera disposition, coordinates, and orientation for each user within the virtual environment need to be considered.

Some of these parameters are not well supported by existing standards. Aiming at reducing both size and complexity of exchanged session descriptions, a dedicated conference configuration server which carries out a preconfiguration step in advance to the offer/answer exchange has been included. The pre-configuration mechanism has two steps: user input and conference event generation and distribution. The first step is performed using a web form for a user to introduce basic information about the session, such as the number and Uniform Resource Identifiers (URI) of participants, the type of VISION scenario to be used and, when needed, user positioning around a shared virtual table and roles performed by each session participant. The server organizes schedules of the participating users and searches for available time slots to suggest a feasible date for the session. Once the user has introduced the required information, the server creates a new conference, assigning to it a conference URI, and generates a VISION conference event document. This XML document is based on an extension of the conference event model, defined in [27]. Under this model, participants in a multi-party session subscribed to a conference event server receive periodic conference events, i.e. XML documents containing information about the session, users, and media being exchanged. New fields conveying VISION-specific information have been added to specify the user position and orientation in a virtual environment, type of VISION scenario associated with the session, or QoS parameters for a media stream.

Standard XML fields are also used to indicate the number and URIs of participating users, their roles in a session, and the list of exchanged media streams along with labels to identify them. An example event showing a session with a single user receiving one media stream is shown in Fig. 4 where VISION-specific parameters are highlighted.

VISION conference events inform users' terminals participating in a given session about media streams sent and received as well as other parameters required to configure the conference and render the 3D scene. This information is used during SIP session initiation to help UAs generating their session descriptions. For illustrative purposes, the UA at the trainer's site in the entertainment scenario would know that 23 media flows need to be transmitted, whereas the one at a trainee's site would send only 4 flows.

Conference event subscriptions involve SIP SUBSCRIBE messages to be sent to the conference URI once a session has started. However, in our implementation, participants receive a conference event before joining the conference. To achieve this, the AS sends SIP REFER messages to each participating UA. Although REFER requests are commonly used in call control scenarios to indicate the recipient to send a SIP INVITE message other request methods such as SUBSCRIBE are also supported. The signaling to distribute VISION conference events is shown in Fig. 5. The

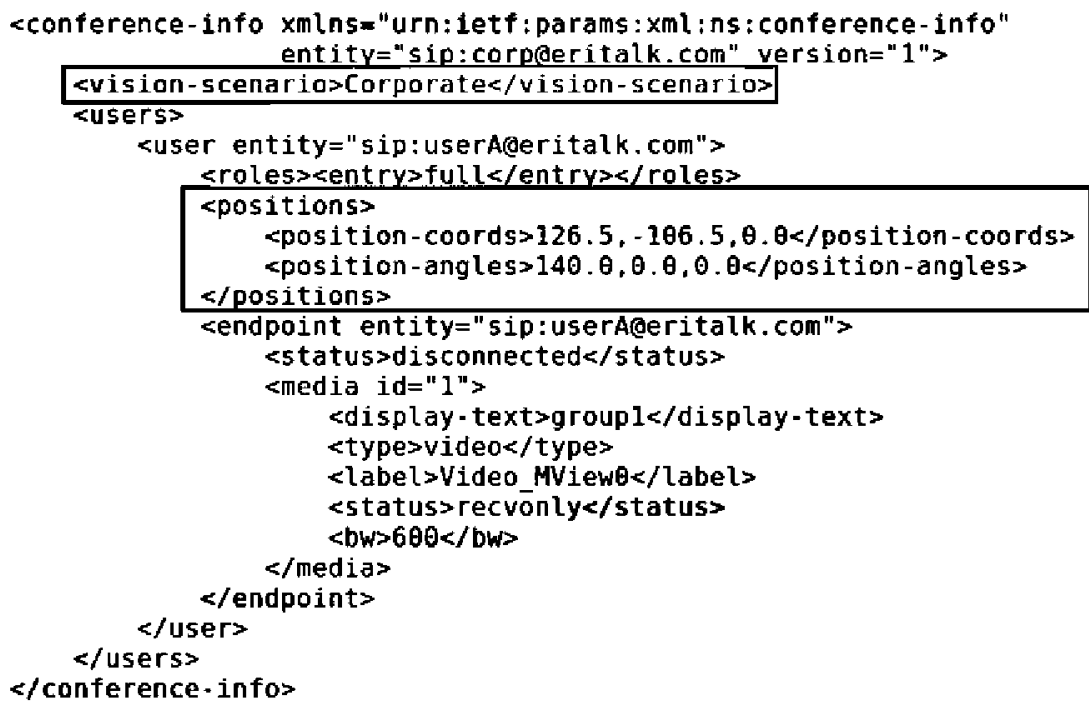

Fig. 4. XML example used in VISION conference events. 


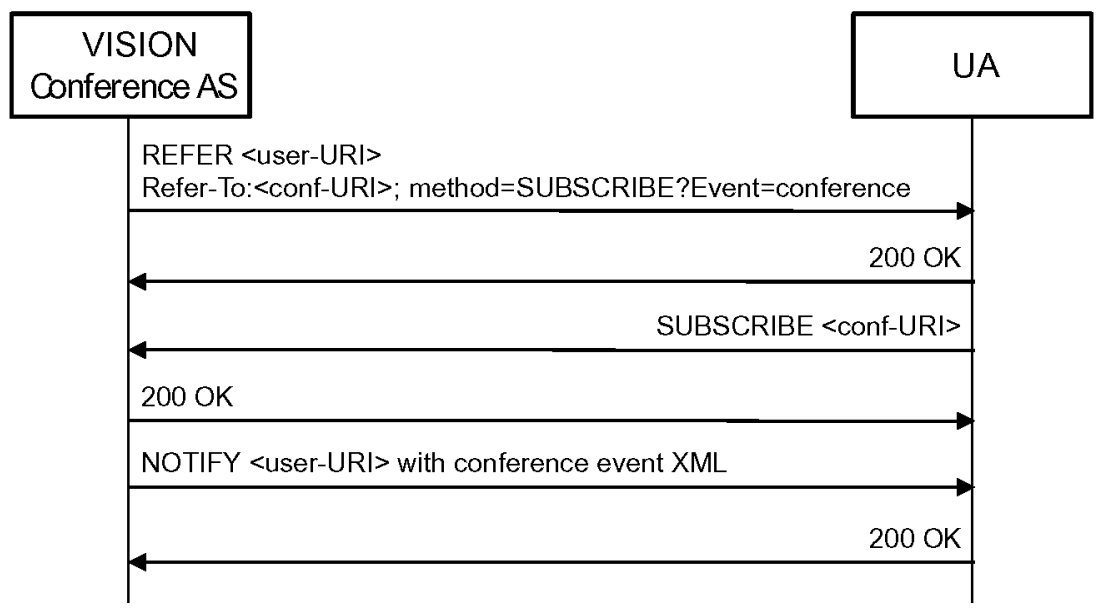

Fig. 5. Conference event-related signaling.

VISION conference AS sends a SIP REFER message, where the Refer-To header points to the conference URI, and includes the method parameter indicating the UA to send a SUBSCRIBE request, as well as a header field specifying that the subscription corresponds to the conference event package. After the REFER transaction is complete, the UA sends the subscription and receives a conference notification.

Once the conference events have been distributed, the configuration server asks users to join the conference by sending SIP REFER requests to their UAs. If the scheduling option is activated, the session will not start until the assigned date. In this case, the server reminds users in advance using SIP-based instant messaging or SMSs. After a pre-determined duration the server automatically terminates the session using a new REFER transaction.

\subsection{Proposed network architecture}

Multimedia communications impose strict requirements on the network to ensure that multimedia flows conveyed over the network can be decoded and smoothly reproduced at reception. This requirement is even more stringent in the VISION project since, as specified in Annex 1 , we deal with a large number of broadband flows. Therefore, advanced mechanisms are needed to guarantee the desired QoS for those flows, specified in terms of packet loss, delay, etc. These advanced mechanisms should be based on protocols and algorithms at the IP layer and even at lower layers, such as at the optical layer.

Strong integration among network layers is actually required to extend QoS provisioning beyond the IP layer. In this regard, we designed the transport network as a set of IP sub-networks connected through a core WSON. In the domain of the access networks the Gigabit-capable Passive Optical Network (GPON) technology [28] was used to implement a FTTH solution. GPON can provide current and future services to users' premises over an optical fiber. A GPON network consists of an Optical Line Terminal (OLT), installed at the operator's premises, and of an Optical Networking Terminal (ONT) at the user's. OLTs contain a number of GPON line ports, each supporting several ONTs connected through a passive optical network. GPON supports asymmetrical bitrates of $2.5 \mathrm{Gbps}$ downstream and 1.25 Gbps upstream; up to $100 \mathrm{Mbps}$ downstream per user could be provided with a very low oversubscription ratio.

When the project started to define the architecture to control such a network, some topics were not fully covered in the standards. In particular, mechanisms to control the core network were only mentioned in TISPAN but not developed to any extent. In view of that, we proposed the hybrid 3GPP/TISPAN architecture shown in Fig. 6 to control the network under consideration. Aiming at integrating IP and WSON, two new functional blocks were defined: the Optical Core Resource Admission and Control Function (OC-RACF) and the Optical Core Border Control Function (OC-BCF). OC-RACF behaves similar to A-RACF; it receives IP resource reservation requests from the SPDF module, verifies reservation availability against the network operator policies, and communicates these requests to the OC-BCF module through the newly defined Re' interface. Additionally, $\mathrm{OC}-\mathrm{BCF}$ is in charge of the optical resources in the optical aggregation node mapping IP flows into optical connections.

The Resource Control Enforcement Function (RCEF) module is connected to A-RACF via the Re interface, being responsible for applying operator-defined QoS policies. It consists of three main modules. First, the configuration manager module which manages IP router's resources, accepting or denying new bandwidth requests according to a priority algorithm. Second, the QoS policies manager module that receives control messages arriving through the Re interface and processes them before handling the contained data to the configuration manager. Third, the hardware access module which accesses router's physical registers. Without loss of generality, a set of four prioritized service levels has been defined. Each service level is labeled with an integer number, being " 1 " the one with the highest priority. Each flow within a session could involve different priority levels.

Regarding the WSON domain, an overlay architecture is proposed, where OC-BCF requests the WSON domain optical connections set up or tear-down through the UNI, as 


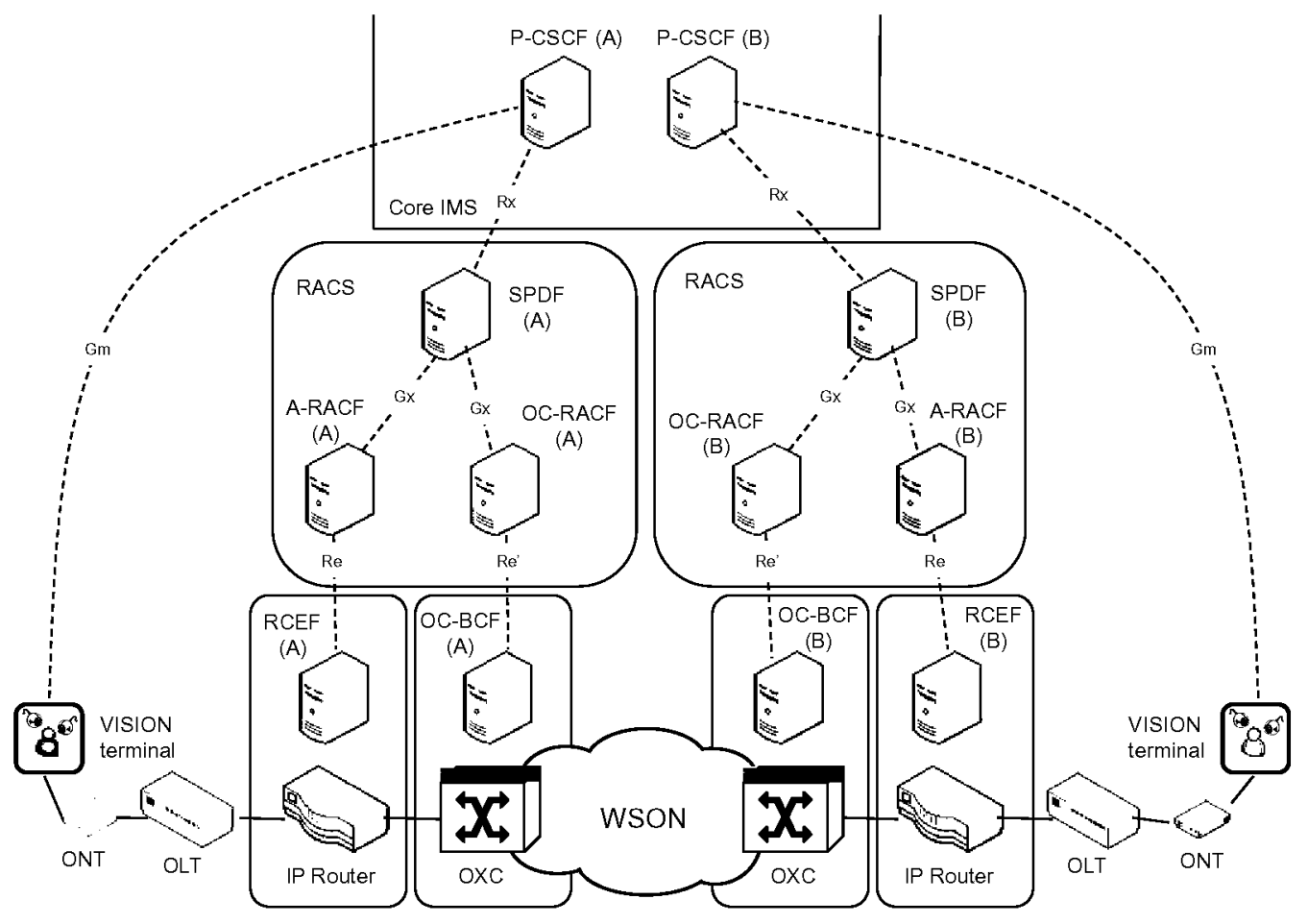

Fig. 6. A general scheme of the VISION network architecture.

illustrated in Fig. 7. It is worth noting that no iteration between $O C-B C F$ and $O X C$ is allowed. Instead, $O C-B C F$ requests arrive to the local Optical Connection Controller (OCC) through the UNI. Next, OCC starts a GMPLS signaling phase using the Internal Network-Network Interface (I-NNI) and sends control commands to the local OXC through the Connection Controller Interface (CCI).

In the course of the project, the proposed architecture has been revised against the emerging standards with the objective of being as aligned as possible with them. However, some minor differences still remain. Apart from the

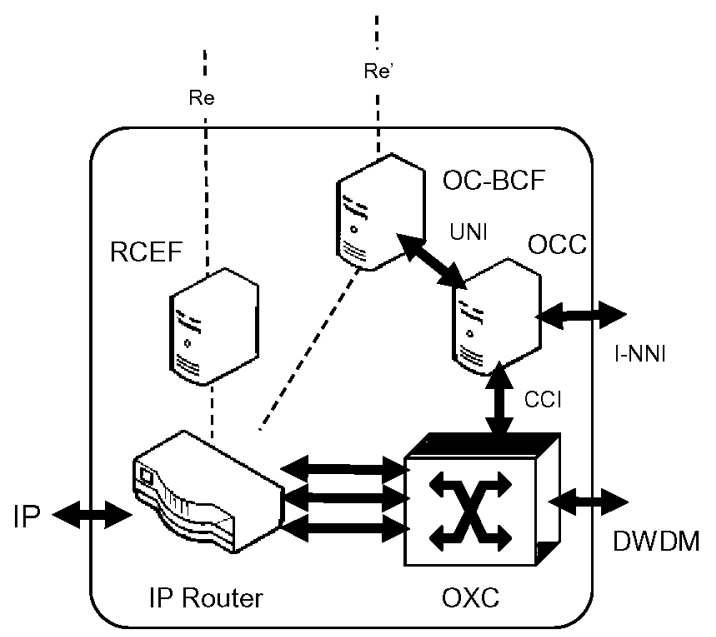

Fig. 7. Overlay architecture using the UNI interface. name of some blocks, TISPAN uses RCEF to denote the enforcement modules in all the network segments while OC-BCF was used in VISION for the optical core; core control functionalities in the project are influenced by the circuit switched nature of the optical network. In addition, some simplifications were allowed so the biggest efforts could be concentrated on specific mechanisms to provide QOS. In this regard, we assumed single IMS provider and single SPDF, where the latter is responsible for user-based policy control so policies could be easily shared and be common for all network segments. Thus, the sequence of operations for policy rule provisioning involves user registering and A-RACF receiving the users' profiles from the Network Attachment Subsystem (NASS) (not shown in the figures). Right after, the user would request a service to the IMS which, together with the suitable application server, would submit QoS information to SPDF. Then, the SPDF maps policies into a request to be sent to its A-RACF and OC-RACF at the same time. For the sake of brevity, $\mathrm{X}-$ RACF is hereafter used to refer to both A-RACF and OCRACF. It is worth noting that in the TISPAN standards, SPDF does not deal with user-related information, but only with service-related policies, being A-RACF the only one which checks the user profiles from NASS.

\subsection{End-to-end signaling}

Fig. 8 illustrates a simple example of the exchanged SIP and Diameter messages to set up a session between users $A$ and $B$. The process starts with user A requesting a new multimedia session to user $B$; the INVITE B SIP message is sent 
to the local P-CSCF (A). The S-CSCF within the core IMS looks for user $B$ in its database and forwards the INVITE message to the local P-CSCF (B) in charge of that user. Finally, the message arrives at user $B$. Upon accepting the session, the 200 OK SIP message is sent backwards to user $A$.

To provide QoS multimedia services, each P-CSCF requests resource reservation to the underlying access network for the multimedia session using Rx Authentication Application Request (AAR) and Authentication Application Answer (AAA) messages. The set of IP flows that defines a media component, the media type (e.g., audio, video), the requested bandwidth, and other information is sent to the local SPDF. Then, SPDF uses these data to generate dynamic PCC rules per each media sub-component, as well as to determine their associated QoS, that will afterwards be installed in the enforcement points. These rules are dynamically generated and/or modified by the SPDF during the session upon reception of Rx messages. Next, SPDF sends messages containing the PCC rules to be applied at the $\mathrm{x}$-RACF modules through the $\mathrm{Gx}$ interface. The request coming from the Rx interface is mapped to zero, one, or more PCC rules and then packed into a Re-Authentication-Request (RAR) Diameter message. Specifically, a Charging-Rule-Definition attribute-value pair (AVP) is used, as depicted in Table 1. The Re-Authentication-Answer (RAA) Diameter message is used to answer the request.

In this simplified scenario where SPDF concentrates all the policy-related decisions, $\mathrm{x}$-RACF modules act just as bidirectional Diameter proxies, with available capacity to perform value-added functionalities. Downstream, they receive requests from the SPDF module through the Gx interface and forward them to the RCEF/ OC-BCF enforcement module through the $\mathrm{Re} / \mathrm{Re}^{\prime}$ interface, respectively. All Diameter messages are logged into a local database for further inspection. Both RCEF and OC-BCF modules implement a specific interface to convey requests to the underlying IP node. In addition to this, the OC-BCF module implements also the UNI interface to request optical connections to the WSON (see Fig. 7).

Upon the reception of a message for a new session, the OC-BCF module obtains from its routing table the IP destination addresses of the remote $\mathrm{OXC}$. This module manages information related to the already established optical connections and their unused bandwidth. Depending on the requested bandwidth, as well as on the available bandwidth of the already established optical connections between the same end-points, the OC-BCF module decides whether to aggregate the flows of the requested session

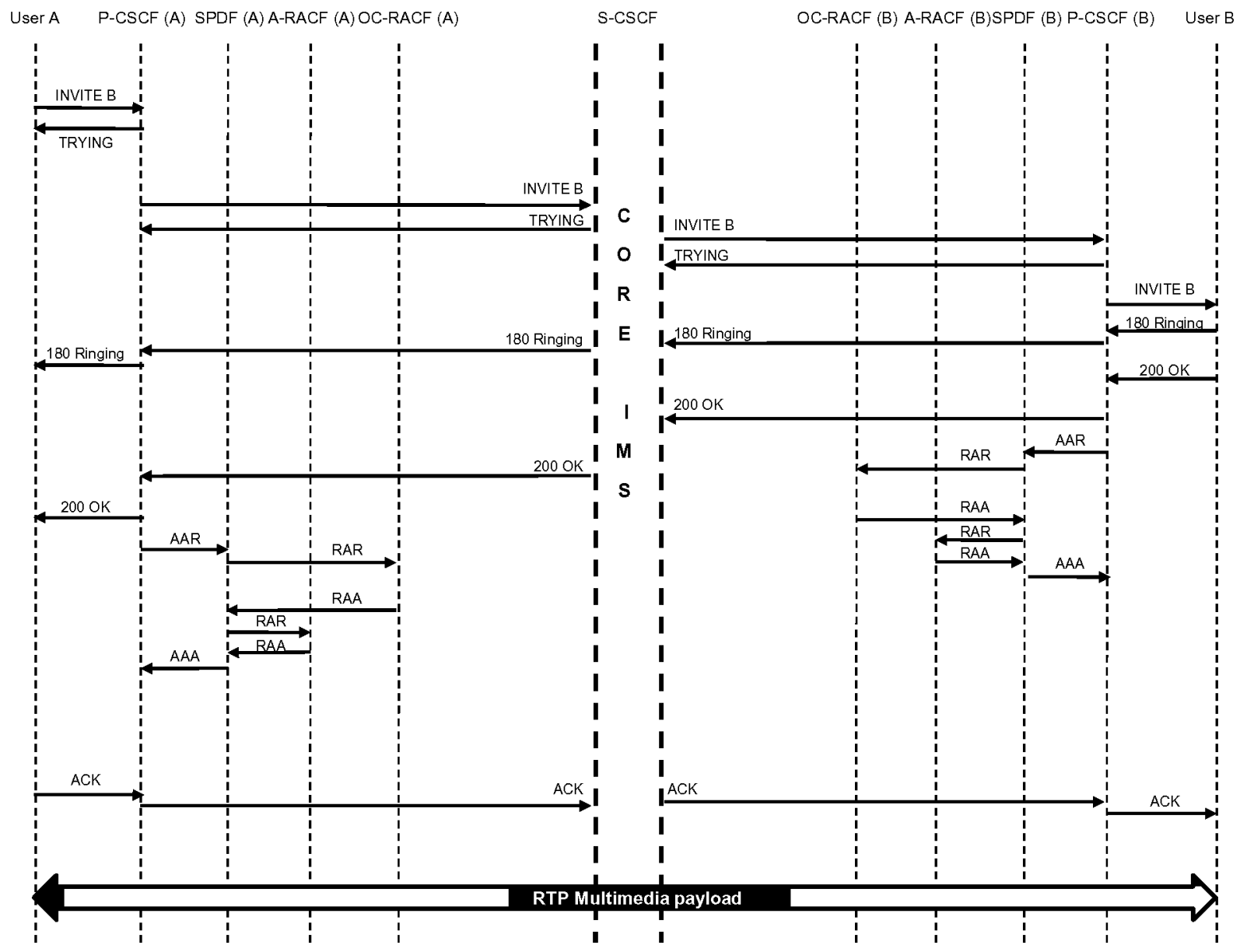

Fig. 8. Setup signaling of a multimedia session with QoS requirements. 
Table 1

Gx RAR Diameter message and its attributes.

\begin{tabular}{ll}
\hline AVP & Function \\
\hline Session-id & Session id provided by SPDF \\
Auth-application-id & Application identify provided by SPDF \\
Origin-host & Origin host of the Diameter message \\
Origin-realm & Origin realm of the Diameter message \\
Destination-realm & Destination realm of the Diameter message \\
Destination-host & Destination host of the Diameter message \\
Re-auth-request-type & Diameter RA type \\
Charging-rule-install & Charging policy to be installed in each x-RACF \\
Charging-rule-definition & Defines the policy to be applied in a flow \\
$\quad$ Charging-rule-name & Contains the name of the charging install policy \\
Flow-description & Defines a filter for a certain IP flow \\
QoS-information & Contains a the QoS policy information \\
QoS-class-identifier & Identifies QoS parameters defined in the charging policy \\
Max-requested-bandwidth-UL & Maximum bandwidth in the upstream flow \\
Max-requested-bandwidth-DL & Maximum bandwidth in the downstream flow \\
Charging-rule-remove & Charging policy to be removed in each x-RACF \\
Charging-rule-definition & Defines the policy to be applied in a flow
\end{tabular}
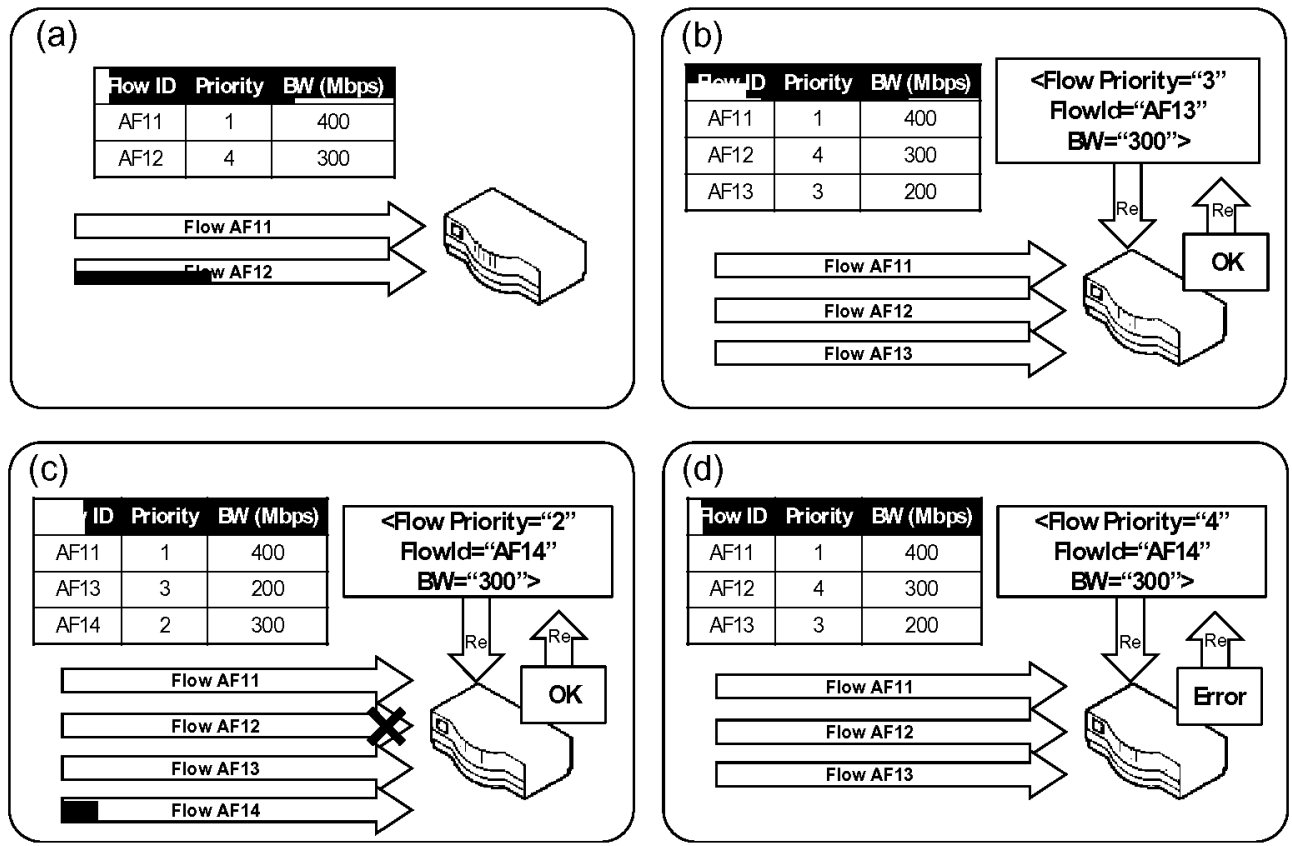

Fig. 9. Example of QoS reservation for a 1 Gbps link.

to an existing optical connection, thus saving optical resources.

Let us assume that no optical connection exists between the optical network end-points. In such case, a new optical connection has to be set up in the WSON and thus, the OC$B C F$ module sends a request through the UNI interface. The request includes the $O X C$ end-points, the required bandwidth, and the desired QoS. After the optical connection is established only the originally requested bandwidth is assigned, remaining the rest unused, ready to be assigned to future end-to-end sessions.

Finally, the request arrives at the RCEF, where QoS policies take care about bandwidth guarantee. In case the required resources are available, changes at the IP router will be accordingly commanded so to reserve resources for the given flows. Otherwise, lower priority flows belonging to different sessions will be discarded. Fig. 9 illustrates this process. Fig. 9a shows the resources reserved for two flows in a $1 \mathrm{Gbps}$ link of a router. Each flow has different bandwidth requirements and different assigned priority. A new QoS request arrives at RCEF in Fig. 9b through the Re interface. Since there are enough resources, RCEF reserves those requested for the new flow. Starting from that state, i.e. only $100 \mathrm{Mbps}$ remain unassigned; Fig. $9 \mathrm{c}$ and d depict two opposite cases. In Fig. 9c a new QoS request with priority 2 arrives at RCEF. Since there are lower priority flows with enough aggregated bandwidth for the new one, reserved resources for flow AF12 are released and the request is accepted. In that case, a notification is sent upwards warning about AF12 preemption. In contrast, 


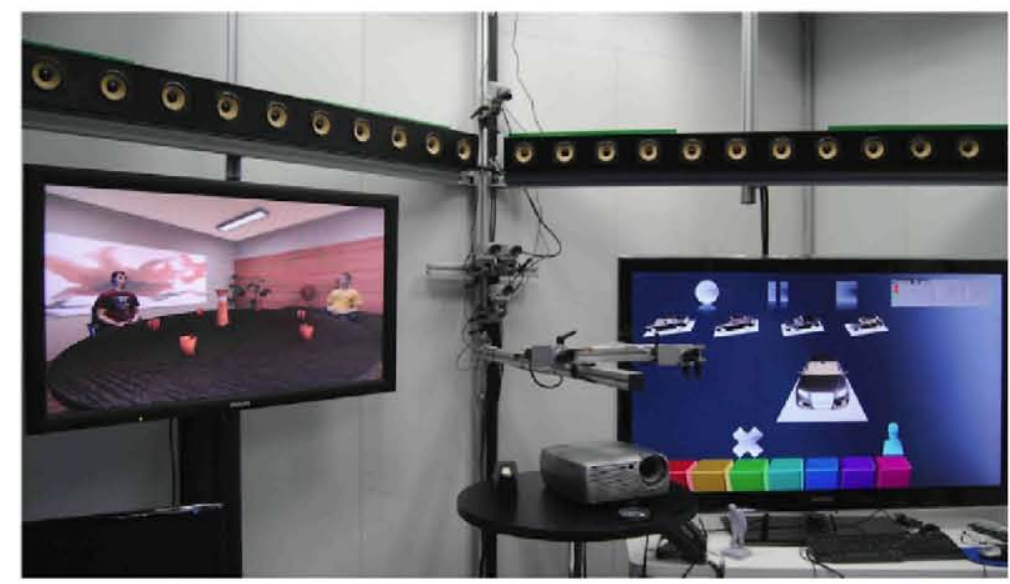

Fig. 10. Example of the corporate scenario.

the request in Fig. 9d is for a flow with priority 4. In that case, no lower priority flows can be preempted and thus RCEF rejects the request.

\section{Experimental deployment and results}

\subsection{Experimental deployment}

The VISION project experimental test-bed was deployed in a distributed manner; equipments in the transport network, video rooms and applications were installed in the Telefónica I+D premises in Barcelona [29], while the core IMS and SPDF modules where kept in the Ericsson's premises in Madrid [30]. Finally, $x$-RACFs and OC-BCFs ran in the UPC's premises in Barcelona [31]. For the test-bed, the control plane was based on a commercial core IMS from Ericsson whereas IP routers were implemented using specific OXC's pluggable cards. Both, GPON and OXC nodes were supplied by Telnet-RI [32]. Fig. 10 illustrates the immersive teleconference in the corporate scenario and Fig. 11 shows the physical layout of ONTs and OXC nodes. Note that OXCS include IP routing and OLT cards in the same chassis.

The SIP user agent was written in Java using [33], an open source Java implementation of the SIP protocol stack. The VISION conference was also written in Java, based on the open source application server GlassFish/Sailfin [34], and deployed as an IMS AS. Functional blocks X-RACF and OC-BCF were implemented as multithread applications in Java and Re' and Gx interfaces were based on JavaDiameterPeer [35], an open-source Java implementation of the Diameter base protocol. Finally, the RCEF module running inside IP routers was developed in C. The next subsections detail the experimental tests performed.

\subsection{Quality of service assessment}

Some tests were performed using the test-bed to experimentally assess the capability of the proposed architecture and QoS mechanisms for the considered scenarios. To this end, two VISION terminals, user A and user B, located in contiguous rooms were connected to the

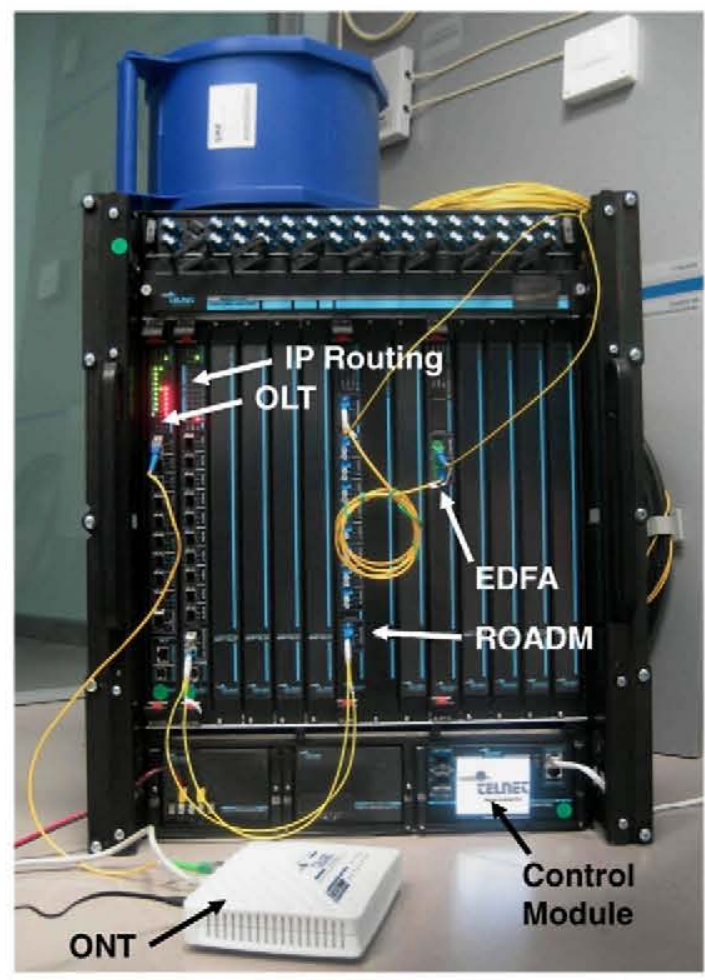

Fig. 11. Physical layout of the experimental nodes.

network in a similar scenario to that of the Fig. $6.18 \mathrm{~km}$ optical coils were used to connect OXC nodes in the core network. Additionally, a Gigabit Ethernet generator/ analyzer was connected to one end of the transport network to inject random traffic for some of the tests. It is worth mentioning that, although all media flows in the considered scenarios are forwarded through a MCU, in these tests we consider point-to-point flows so to better characterize the transport network.

Several tests related with QoS assessment were performed. Firstly, we analyzed the QoS degradation when the reserved bandwidth is reduced emulating competition among flows of different sessions. Here it is worth 


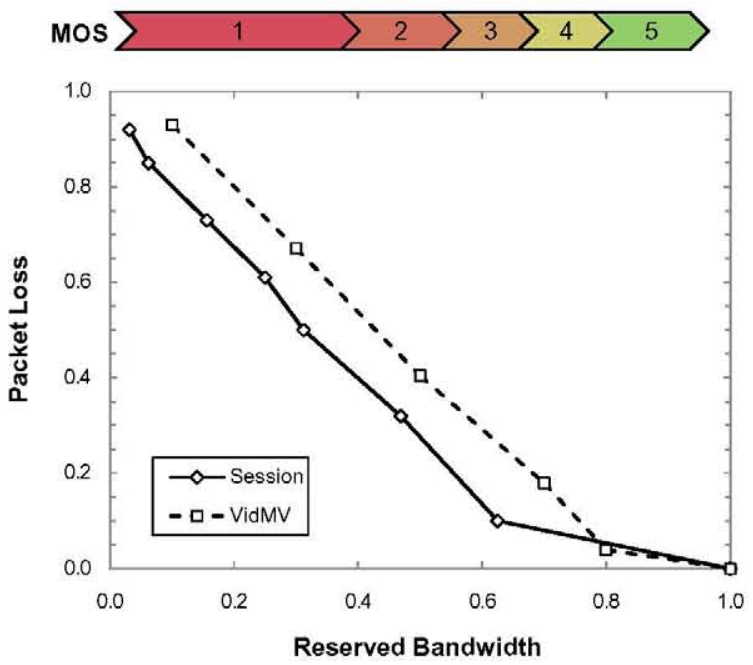

Fig. 12. Packet loss against the reserved bandwidth for all flows belonging to a single session and for one of the video signaling flows within a session (VidMV). The observed MOS is also shown.

highlighting that VISION scenarios exchange video flows coded as specified in [21], which are encapsulated into RTP payloads and delivered over the network. As a consequence of this, each image frame is fragmented into several IP packets, and thus the loss of a single packet may entail the loss of an entire image frame. While the application loses image frames, the user perceives that the videoconferencing service is not delivered correctly. The obtained behavior is represented in Fig. 12 for a whole session and for a single video signaling flow in normalized values. As expected, in the presence of congestion, packet loss increases when the guaranteed bandwidth for a session is reduced. However, as soon as packet loss exceeds from $5 \%$ the user perceived quality degrades sharply. This is even more evident for particular flows as it is the case of video signaling flows.

Aiming at quantifying the quality perceived by the users when the available bandwidth is reduced, we used the Mean Opinion Score (MOS) criterion [16], where the perceived quality is quantified ranging from 1 ("Bad") to 5 ("Excellent"). As shown in Fig. 12, when the available bandwidth is lower than $70 \%$ with respect to the required one, the perceived quality is poor, and only when it is over $90 \%$ users perceive good quality, as a result of error correction performed by the decoder. These results enforce the need of end-to-end resource reservation preventing sessions to compete for shared resources. In addition to this, since all flows are equally important in a session (e.g., if all video flows experience no packet losses but audio quality is bad, the whole session becomes useless) no difference can be done in terms of priority. In view of this, we propose to use per session/per service priority policies, assigning the highest to the corporate, an intermediate to the entertainment, and the lowest to the residential services.

Secondly, we measured end-to-end packet delay in accordance with [36]. Fig. 13 plots the obtained maximum and on average delay values as a function of the packet size. As result of the state-of-the-art technologies used in

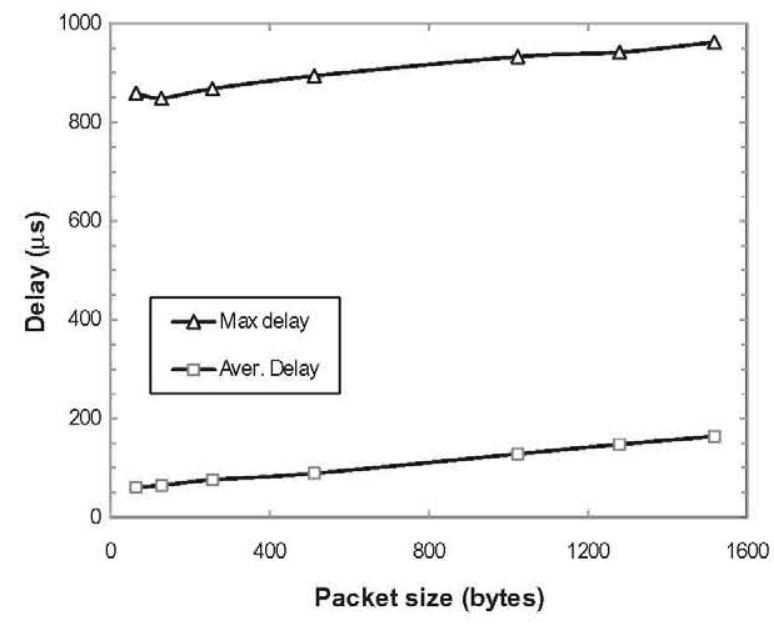

Fig. 13. Delay against packet size.

the network, delay is under $1 \mathrm{~ms}$ in all cases i.e., only $1 \%$ of the maximum specified [37] which includes also coding/decoding stages [38]. Therefore, the network-produced delay does not significantly impact the end-to-end delay and as a consequence, no effect is observed by the users reinforcing thus real-time user experience.

\subsection{End-to-end signaling performance evaluation}

For illustrative purposes, next figures reproduce in part the signaling messages exchanged to set-up a new session in the simplest residential scenario. Fig. 14 shows a fragment of the session description included in the initial SIP INVITE request. In this scenario, each user agent sends and receives four media flows. User agents determine the number and type of flows to be used in the selected scenario from the conference XML file received previously to the session initiation, as described in Section 3. Fig. 14 depicts the parameters used for the downlink video flow being the remaining flows very similar to this. The media information includes the media type, codec configuration, listening port and address, and bandwidth requirements. In addition, each flow is labeled for its identification, which is particularly important for the more complex scenarios where multiple video and application data flows are used.

Fig. 15 illustrates a RAR message sent by the SPDF block to one of the OC-RACFs through the Gx interface. Message contents are as specified in Table 1. As shown, four different rules need to be installed, one per each multimedia flow. $4 \mathrm{Mbps}$ for video flows are requested for both upstream and downstream directions within the first rule and assigned the lowest priority class (4). End users IP addresses and ports are also provided.

Regarding session control, recall that two key features characterize our approach: the initial media configuration phase and the size and complexity of the session description messages exchanged as a consequence of the number of media streams. Since these features may have a negative impact on session system scalability and control delay, a number of load tests have been performed to quantify this impact. To this end, an automatic tool that generates 


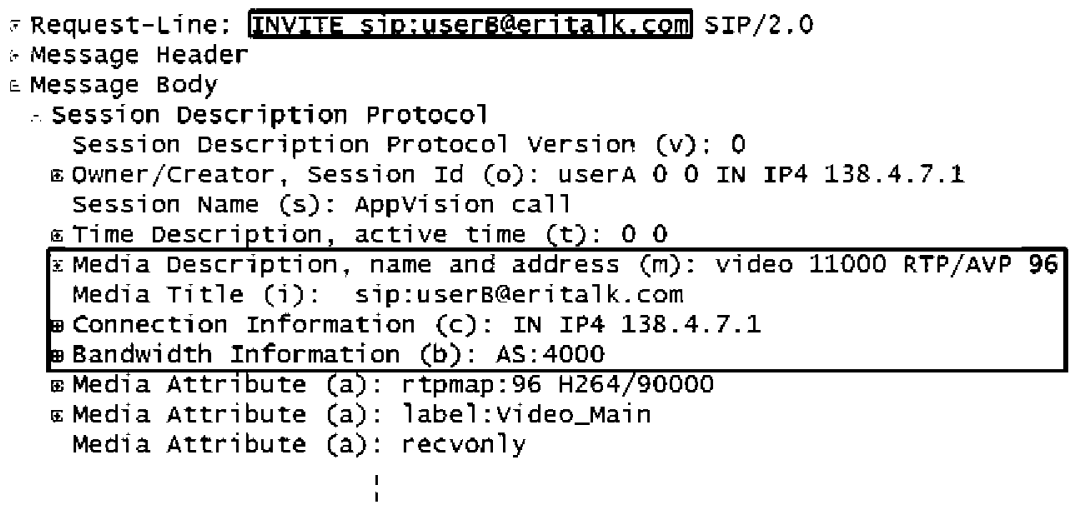

Fig. 14. SIP INVITE request and session description used to set-up a new session.

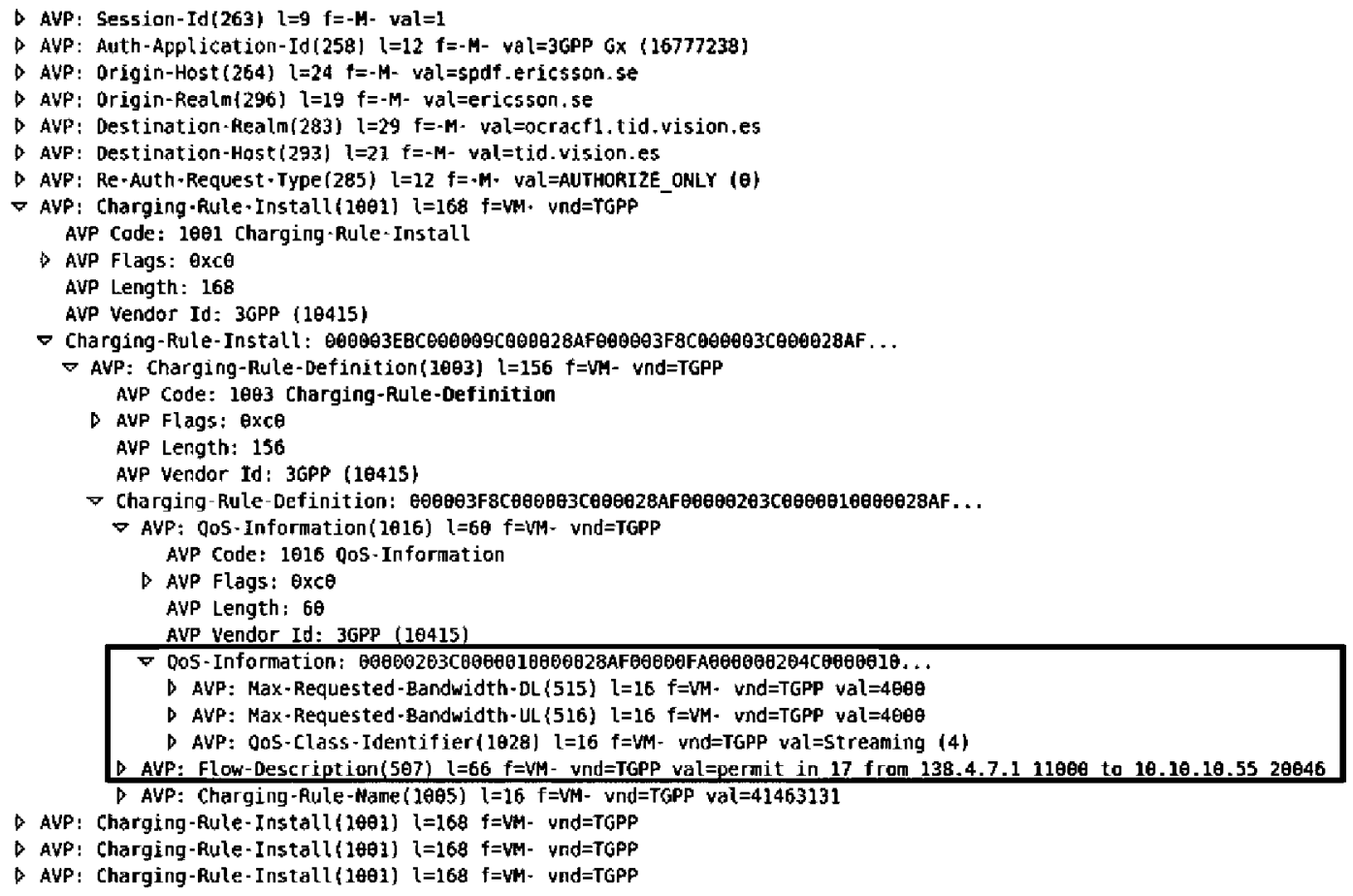

Fig. 15. RAR message.

requests for the definition of new conferences in the AS was used. For each test, CPU and memory consumption statistics were stored for the VISION conference AS, which run on a $2.1 \mathrm{GHz}$ Intel Core $2 \mathrm{CPU}$ with $3 \mathrm{~GB}$ RAM memory running Windows 7 . In view that the performance is highly dependent on the number of users in a session, we performed tests involving 2 and 4 users per conference.

Figs. 16 and 17 focus on scalability. Starting from a request rate of 1 request/s, the rate was steadily increased with a slope of $5 / 3$ requests/s until the CPU or memory usage were exhausted. Upon receiving a request, the AS performs, for each user in the session, the signaling exchange described in Fig. 5. The UAs were set up to automatically accept REFER requests and immediately perform conference event subscriptions, minimizing the initial configuration time. Fig. 16 shows the CPU load against the request rate for setting-up a conference for the considered scenarios. As observed, requests rates as high as 93 for the complex corporate, 144 for the entertainment, and even 157 for the residential scenario can be served with CPU usage under 90\% when conferences involve 2 users. Nevertheless, as a result of that larger XML files need to be exchanged when conferences involve 4 users, requests rates drop to one third of the above values, being 33, 54, and 59, respectively. Fig. 17 shows the memory usage increment against the request rate. Although memory usage also depends on the scenario, request rate are not limited by this fact, in contrast to the CPU load.

Next, the configuration delay, defined as the time from the reception of a session creation request at the AS and the successful delivery of a conference event for every user involved in that session, was measured. Note that the initial 


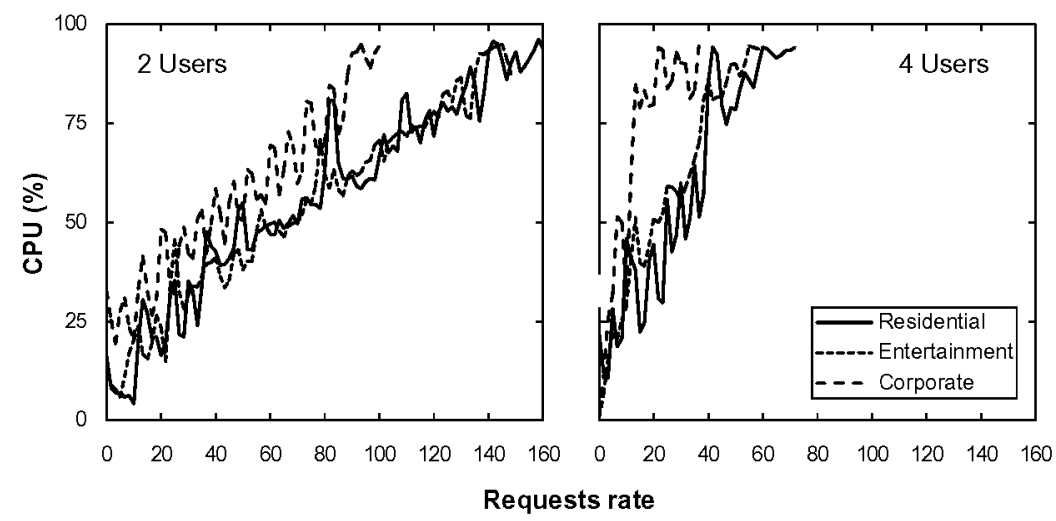

Fig. 16. CPU load as a function of the request rate (requests/s) for sessions with 2 (left) and 4 (right) users.

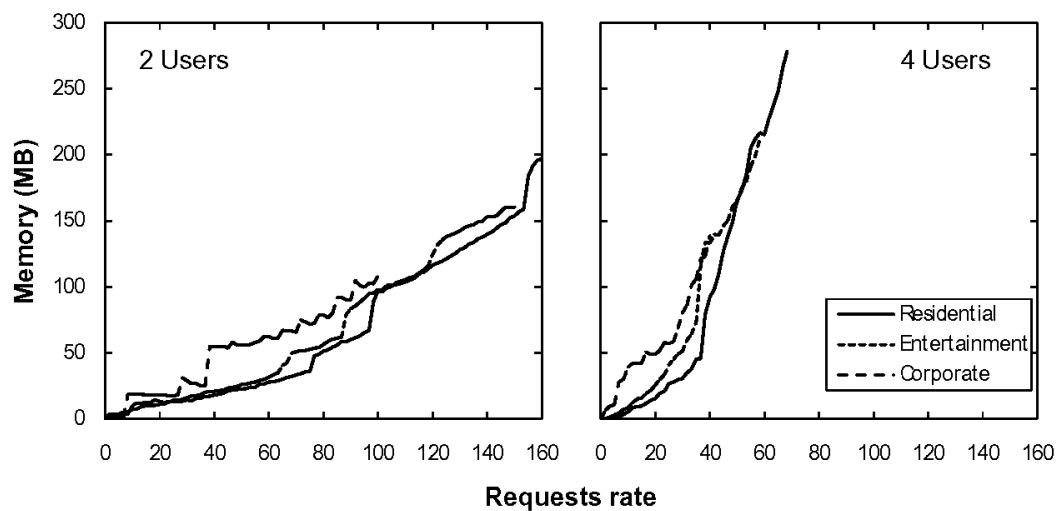

Fig. 17. Memory usage increment as a function of the request rate for sessions with 2 (left) and 4 (right) users.

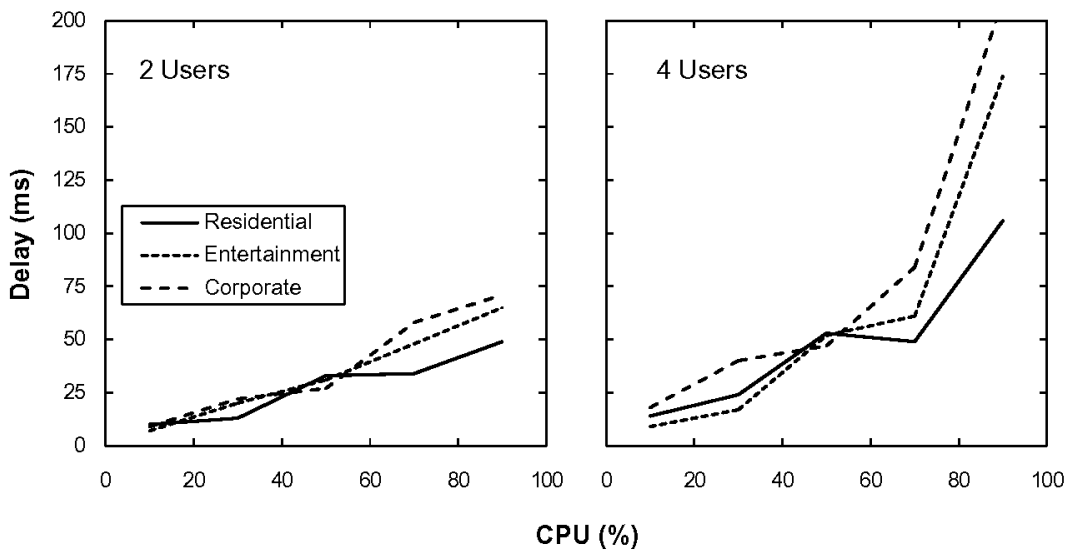

Fig. 18. Initial configuration delay as a function of the CPU load for sessions with 2 (left) and 4 (right) users.

configuration represents the most important overhead introduced by the SIP signaling in our approach. Fig. 18 shows the introduced delay as a function of the CPU load, where each value represents the average of 100 results. Delays lower than $70 \mathrm{~ms}$ on average ( $120 \mathrm{~ms}$ in the worst case) when 2 users are involved rising to $200 \mathrm{~ms}$ ( $350 \mathrm{~ms}$ in the worst case) when involving 4 users were obtained for high CPU loads. Notwithstanding, those delays are completely negligible when compared to total set-up delays, which include QoS resource reservation and optical connection set-up, presented next. It is worth mentioning that in our tests all users were in the same location and then measured delays do not include any propagation delay.

Finally, we measured both session set-up and release times in the test-bed, assuming both the worst case scenario where optical connectivity set-up was needed and 
Table 2

Total set-up time (seconds) analysis.

\begin{tabular}{llll}
\hline Scenario & $\begin{array}{l}\text { QoS resource } \\
\text { reservation }\end{array}$ & $\begin{array}{l}\text { Optical connection set-up } \\
\text { +flow aggregation }\end{array}$ & $\begin{array}{l}\text { Set-up } \\
\text { time }\end{array}$ \\
\hline Residential & 1 & 2 & 2 \\
Entertainment & 5.75 & 2 & 5.75 \\
Corporate & 5.75 & 2 & 5.75 \\
\hline
\end{tabular}

Table 3

Residential scenario.

\begin{tabular}{llll}
\hline Direction & $\begin{array}{l}\text { Aggregated } \\
\text { flow }\end{array}$ & $\begin{array}{l}\text { Number } \\
\text { of flows }\end{array}$ & $\begin{array}{l}\text { Total } \\
\text { bandwidth } \\
\text { (Mbps) }\end{array}$ \\
\hline A $\rightarrow$ B 4 flows (4.8 Mbps) & Video & 1 & 4.0 \\
& Audio & 2 & 0.3 \\
& Photolog & 1 & 0.5 \\
B $\rightarrow$ A 4 flows (4.8 Mbps) & Video & 1 & 4.0 \\
& Audio & 2 & 0.3 \\
& Photolog & 1 & 0.5 \\
\hline
\end{tabular}

Table 4

Entertainment scenario.

\begin{tabular}{llll}
\hline Direction & $\begin{array}{l}\text { Aggregated } \\
\text { flow }\end{array}$ & $\begin{array}{l}\text { Number } \\
\text { of flows }\end{array}$ & $\begin{array}{l}\text { Total } \\
\text { bandwidth } \\
\text { (Mbps) }\end{array}$ \\
\hline A $\rightarrow$ B 4 flows (4.8 Mbps) & Video & 1 & 4.0 \\
& VidMV1 & 6 & 4.8 \\
& VidMV2 & 3 & 2.4 \\
& VidMV3 & 3 & 2.4 \\
& VidMV4 & 6 & 4.8 \\
& Voxel & 1 & 0.02 \\
& Audio & 2 & 0.3 \\
& Photolog & 1 & 0.5 \\
B A 4 flows (4.8 Mbps) & Video & 1 & 4.0 \\
& Audio & 2 & 0.3 \\
& Photolog & 1 & 0.5 \\
\hline
\end{tabular}

Table 5

Corporate scenario.

\begin{tabular}{rlll}
\hline \multicolumn{1}{l|}{ Direction } & $\begin{array}{l}\text { Aggregated } \\
\text { flow }\end{array}$ & $\begin{array}{l}\text { Number } \\
\text { of flows }\end{array}$ & $\begin{array}{l}\text { Total } \\
\text { bandwidth } \\
\text { (Mbps) }\end{array}$ \\
\hline A $\rightarrow$ B 4 flows & Video & $\mathbf{1}$ & 4.0 \\
(4.8 Mbps) & VidMV1 & 6 & 4.8 \\
& VidMV2 & 3 & 2.4 \\
& VidMV3 & 3 & 2.4 \\
& VidMV4 & 6 & 4.8 \\
& Voxel & $\mathbf{1}$ & 0.02 \\
& Audio & 2 & 0.3 \\
& Photolog & $\mathbf{1}$ & 0.5 \\
B $\rightarrow$ A 4 flows & Video & $\mathbf{1}$ & 4.0 \\
(4.8 Mbps) & VidMV1 & 6 & 4.8 \\
& VidMV2 & 3 & 2.4 \\
& VidMV3 & 3 & 2.4 \\
& VidMV4 & 6 & 4.8 \\
& Voxel & $\mathbf{1}$ & 0.02 \\
& Audio & 2 & 0.3 \\
& Photolog & $\mathbf{1}$ & 0.5 \\
\hline
\end{tabular}

low load conditions. Total set-up times for all three scenarios ranged from about 2.5 to $6 \mathrm{~s}$ whereas tear-down times were about $2.5 \mathrm{~s}$. Note that the set-up time threshold for voice services is $7.5 \mathrm{~s}$ [39].
Aiming at performing an in depth analysis of the set-up times, we measured the contribution of the QoS resource reservation and the optical connection set-up and flow aggregation over the total set-up time for every single scenario. Table 2 shows the measured times. It is worth highlighting that total set-up times are given by the maximum of the QoS resource reservation process carried out by RCEF modules and the optical connection set-up performed by OC-BCF modules, since both processes are performed in parallel. All the same in the entertainment, like in the corporate, resource reservation took $5.75 \mathrm{~s}$ as a consequence of the large number of flows involved. On the opposite, in the residential scenario resource reservation took only $1 \mathrm{~s}$. Regarding flow aggregation and optical connection set-up, only $2 \mathrm{~s}$ are needed.

\section{Conclusions}

This paper showed the network architecture developed within the Spanish-funded VISION project. The inclusion of novel functional blocks dedicated to control the optical core network and the redefinition of existing ones allow prioritizing multimedia flows guaranteeing QoS to provide future multimedia services such as tele-presence and 3D videoconferencing services. Moreover, extensions to the conference event model have been proposed to support future QoS demanding multimedia services.

An experimental integrated access and core converged network architecture have been deployed to provide the requested QOS to end-to-end IP sessions, experimentally assessing the proposed control plane to provide real-time 3D based videoconferencing services. End-to-end session set-up and release has been demonstrated and the quality perceived by the users quantified using the MOS criterion. Per-session/per service priority policies were proposed to enhance preemption management. Network delay proved to be negligible as a consequence of the technology used at the access and at the core networks. Moreover, the scalability of the proposed signaling architecture was demonstrated, and low signaling delays were shown. As a consequence, session set-up and release times were under the recommended threshold for voice services.

As a final conclusion, the designed network architecture together with the technology and the proposed priority policies allow providing future QoS-demanding multimedia services.

\section{Acknowledgments}

The authors would like to acknowledge the help from several people during the course of this project. In particular, Francis Rastrilla and Héctor Fernández from TELNET-RI for their contribution in the development of the network equipments specially designed for VISION project and Ana Sanz from Ericsson for helpful discussions regarding network architecture.

This work has been partially supported by the Spanish Administration organism CDTI, under project CENITVISION 2007-2010. 


\section{Appendix A. ANNEX 1. Flow Definition for the considered scenarios}

Tables 3-5.

\section{References}

[1] Vision Project, <http://www.cenit-vision.org/>.

[2] NGN Functional Architecture Release 2, ETSI RES 282 001, 2007.

[3] J. Song, M. Chang, S. Lee, J. Joung, Overview of ITU-T NGN QoS Control, IEEE Communications Magazine 45 (2007) 116-123.

[4] IP Multimedia Subsystem (IMS); Stage 2, 3GPP TS 23.228 , 2011.

[5] Architecture for the automatically switched optical network (ASON), ITU-T G.8080, 2001

[6] E. Mannie, Generalized Multi-Protocol Label Switching (GMPLS) Architecture, IETF RFC 3945, 2004.

[7] G. Swallow, J. Drake, H. Ishimatsu, Y. Rekhter, Generalized Multiprotocol Label Switching (GMPLS), User-Network Interface (UNI): Resource ReserVation Protocol-Traffic Engineering (RSVPTE). Support for the Overlay Model, IETF RFC 4208, 2005.

[8] S. Chatterjee, B. Tulu, T. Abhichandani, H. Li, SIP-Based Enterprise Converged Networks for Voice/Video-Over-IP: Implementation and Evaluation of Components, IEEE Journal on Selected Areas in Communications 23 (2005) 1921-1933.

[9] F. Callegati et al., SIP-Empowered optical networks for future IT services and applications, IEEE Communications Magazine 47 (2009) $48-54$

[10] W. Cerroni, B. Martini, M. Gharbaoui, A. Campi, F. Baroncelli, P. Castoldi, F. Callegati, et al., Experimental validation of a SIP-based Platform for Service Oriented Optical Network, in: Proceedings of OFC/NFOEC, 2010

[11] J. Rosenberg, H. Schulzrinne, G. Camarillo, A. Johnston, J. Peterson, R. Sparks, M. Handley, E. Schooler, SIP: Session Initiation Protocol, IETF RFC 3261, 2002.

[12] P. Agrawal, J. Yeh, J. Chen, T. Zhang, IP multimedia subsystems in 3GPP and 3GPP2: overview and scalability issues, IEEE Communications Magazine 46 (2008) 138-145.

[13] V. Balasubramaniyan, A. Acharya, M. Ahamad, M. Srivatsa, I. Dacosta, C. Wright, Servartuka: Dynamic distribution of state to improve SIP server scalability, in: Proceedings of International Conference on Distributed Computing Systems (ICDCS), 2008.

[14] A. Buono, S. Loreto, L. Miniero, S. Romano, A distributed IMS enabled conferencing architecture on top of a standard centralized conferencing framework, IEEE Communications Magazine 45 (2009) 152-159.

[15] P. Bellavista, A. Corradi, L. Foschini, IMS-based presence service with enhanced scalability and guaranteed QoS for interdomain enterprise mobility, IEEE Wireless Communications 16 (2009) $16-23$.

[16] R. Mok, E. Chan, R. Chang, Measuring the Quality of Experience of HTTP Video Streaming, in: Proceedings of IEEE/IFIP IM, 2011.

[17] Y. Liang, J. Apostolopoulos, B. Girod, Analysis of packet loss for compressed video: effect of burst losses and correlation between error frames, IEEE Transactions on Circuits and Systems for Video Technology 18 (2008) 861-874

[18] R. Stankiewicz, A. Jajszczyk, QoE Assurance in Converged Networks, Computer Networks 55 (2011) 1459-1473.

[19] P. Pérez, N. García, Lightweight multimedia packet prioritization model for unequal error protection, IEEE Transactions on Consumer Electronics 57 (2011) 132-138.

[20] I. Vidal, J. Garcia-Reinoso, I. Soto, F. Valera, Evaluating extensions to IMS session setup for multicast-based many-to-many services, Computer Networks 55 (2011) 600-621.

[21] Advanced video coding for generic audiovisual services, ITU-T H.264, 2009.

[22] J. Rosenberg, H. Schulzrinne, An Offer/Answer Model with Session Description Protocol (SDP), IETF RFC 3264, 2002.

[23] H. Schulzrinne, S. Casner, R. Frederick, V. Jacobson, RTP: A transport protocol for Real-Time Applications, IETF RFC 3550, 2003.

[24] P. Calhoun, J. Loughney, E. Guttman, G. Zorn, J. Arkko, Diameter Base Protocol, IETF RFC 3588, 2003.

[25] Policy and charging control architecture, 3GPP TS 23.203, 2011.

[26] Resource and Admission Control Subsystem (RACS); Functional Architecture; Release 2, ETSI TS 182 019, 2009.
[27] J. Rosenberg, H. Schulzrinne, O. Levin, A Session Initiation Protocol (SIP). Event Package for Conference State, IETF RFC 4575, 2006.

[28] Gigabit-capable Passive Optical Networks (GPON): General characteristics, ITU-T G.984.1, 2008

[29] Telefónica l+D, <http://www.tid.es>.

[30] Ericsson, <http://www.ericsson.com>.

[31] F. Agraz et al., Design and implementation of a GMPLS-controlled grooming-capable optical transport network, IEEE/OSA J. Opt. Commun. Netw., vol. 1, pp. A258-A269, 2009.

[32] Telnet Redes Inteligentes, <http://www.telnet-ri.es/>

[33] MjSip, <http://www.mjsip.org/>

[34] GlassFish/Sailfin, <http://glassfish.java.net/>

[35] JavaDiameterPeer, <http://www.openimscore.org/>

[36] S. Bradner, J. McQuaid, Benchmarking Methodology for Network Interconnect Devices, IETF RFC 2544, 1999.

[37] Internet protocol aspects - Quality of service and network performance, ITU-T Rec. Y. 1541, 2006.

[38] R. Schreier, A. Rothermel, A Latency Analysis on H.264 Video Transmission Systems, International Conference on Consumer Electronics, 2008

[39] Call processing performance for voice service in hybrid IP networks, ITU-T Rec. Y.1530, 2007.

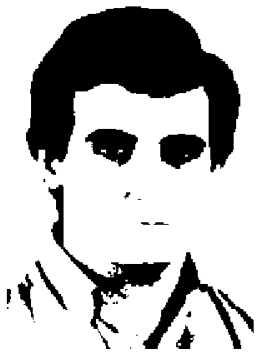

Luis Pérez received the M.Sc. degree in telecommunications engineering from Universidad Politếcnica de Madrid (UPM) in 1983. He joined Telefónica's R\&D department in 1986, where he was involved in a related European project (LION). He worked in the development of a system (MORE) to digitalize the analogical public switching systems (Alcatel PC-1000, Ericsson ARF). From 1997 he has been participating in several National and European projects related to NGN, focusing on IP/MPLS networking and testing, study and evaluation of broadband access technologies (xDSL) and deployment and testing of multimedia applications over IP networks.

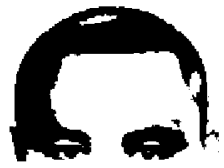

Luis Velasco received the B.Sc. degree in telecommunications engineering from Universidad Politécnica de Madrid (UPM) in 1989, the M.Sc. degree in physics from Universidad Complutense de Madrid (UCM) in 1993, and the Ph.D. degree from Universitat Politècnica de Catalunya (UPC) in 2009. In 1989 he joined Telefónica of Spain and was involved in the specifications and first office application of the Telefónica's SDH transport network. In 2003 he joined UPC, where currently he is an Assistant Professor in the Computer Architecture Department (DAC) and a Researcher in the Optical Communications group (GCO) and the Advanced Broadband Communications Center (CCABA). He has participated in various IST FP-6 and FP-7 European research projects such as EU DICONET, BONE, IST NOBEL 2, ePhoton/ONe+, and STRONGEST. His interests include planning, CAPEX/ OPEX issues, routing and resilience mechanisms in multilayer networks.

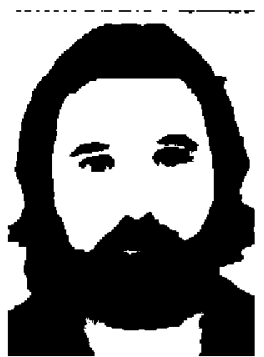

Juan Rodriguez received the Telecommunications Engineering degree from Universidad Politécnica de Madrid (UPM), Spain. He joined Telefónica I+D in 2004, in the "IP Access Network Technologies" Department. There, he was involved in several R\&D European projects, like CELTIC BANITS and FP6 MUPBED. In 2007 he joined Novanotio, working for several network-related Groups within Telefónica $I+D$, where he has continued his R\&D work in projects like CELTIC BANITS2 and R2D2 Networks or CENIT VISION. He has also been involved in several innovation activities for Telefónica of Spain, within the "Photonic Networks" Department. 


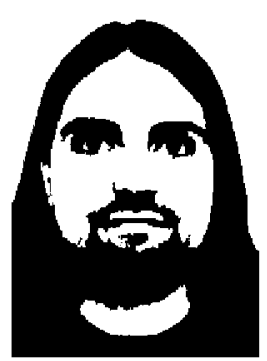

Pedro Capelastegui received the M.Sc. degree in telecommunications engineering from Universidad Politécnica de Madrid (UPM) in 2007, and is currently pursuing his Ph.D. He works in the Telematics Engineering Department at UPM as a researcher. His research interests include IMS, service enablers, and immersive multimedia communications.

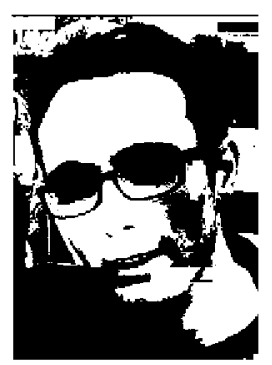

Guillem Hernández-Sola received his M.Sc. degree in telecommunications engineering in 2008 from the Universitat Politècnica de Catalunya (UPC). Currently, he is pursuing his Ph.D. in the Signal Theory and Communications Department (TSC) at UPC. He joined the Optical Communications Group (GCO) in 2008. He is working in several R\&D National and IST FP-7 European research projects like EU DICONET and VISION. His research interests concern multi-domain path computation procedures, PCE architectures in GMPLS, quality of service issues, and future IT services of next-generation optical transport networks.

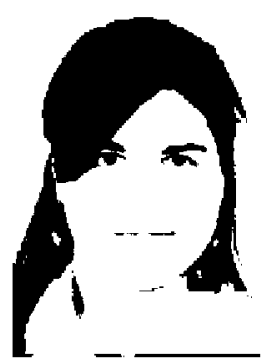

Lorena Calavia received the M.Sc. degree in Telecommunications Engineering by the University of Valladolid (UVA), where she currently works as a researcher and is a Ph.D. candidate. She has been involved in several National and European projects such as OPUCE, VISION, MIO!, WIMSAT and HuSIMS covering topics such as NGNs, contextawareness, service engineering, semantic characterization and inference and applied artificial intelligence.

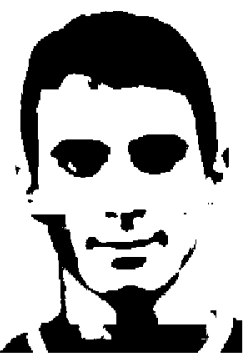

Antonio Marqués received his M.Sc. degree in Telecommunications Engineering in 2006 from Universitat Politècnica de Catalunya (UPC). Currently, he is working in Telefonica R\&D as a senior developer. He has a 5 year experience developing real-time video applications and working with innovative technologies related especially to videoconference systems. For all this time he has been working in several investigation projects financed by different institutions, likke CELTIC TIFANIS, FP7 3DPresence and CENIT VISION. His work area is mainly focused on system's architecture and real-time processing components.

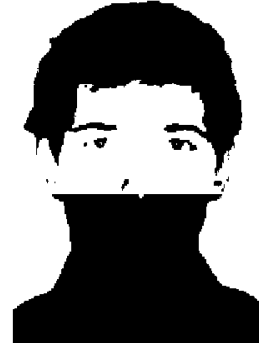

Borja Iribarne obtained his M.Sc. degree in Telecommunications Engineering in 2007 from University of Alcala de Henares (UAH), Spain. He joined Telefónica I+D in 2009 working for both network intelligence networks and digital home initiatives. His activities are focused in NGN/IMS platforms, involved in many research and innovation projects, such as NETLAB (Celtic Programme), VISION (Spanish National Research Programme CENIT), BeFEMTO (Framework Programme VII) and Indoor Connectivity

(Corporative Innovation Project).

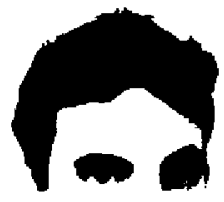

Amador Pozo obtained his B.Sc. degree in Telecommunications Engineering from the Universidad of Zaragoza in 2007. He joined Telnet-RI in 2009, at optical network devices area of the R\&D department. His expertise areas are GPON, GMPLS, Ethernet and related protocols as well as embedded systems and low-level programming. He has also participated in R2D2 and 100GET European research projects.

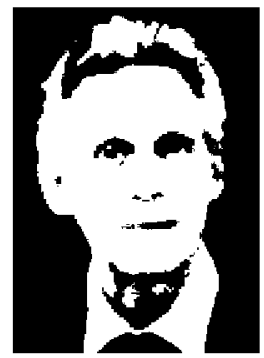

Antoine de Poorter holds a B.Sc. degree in Electrical Engineering from the Higher Technical School in Breda (Netherlands) but he has worked most of his professional life in the telecommunications area. He started working with analogue mobile systems, then the GSM system and later the UMTS system. He has participated in various $\mathrm{FP}-6$ and $\mathrm{FP}-7$ research projects like EUQoS and ADAMANTIUM. His main areas of competence are related to Network User Databases, Mobile network security, mobile Multimedia systems and $3 G$ applications. He currently works in the innovation unit at Ericsson R\&D in Spain. 